\title{
Discapacidad visual. Una experiencia en el profesorado estatal del departamento de Santander, Colombia
}

Volumen 6 N .046 enero - junio de 2019

ISSN: 0122-4328

ISSN-E: 2619-6069 pp. 73-88
Visual Impairment.

An Experience

in Public Service

Teachers of the

Department of

Santander, Colombia
Deficiência visual, uma experiência no corpo docente estadual do departamento de Santander, Colômbia

\section{Luz Mila Duarte Serrano*}

Nury Esther Pabón Bautista*

Angélica María Ascencio Pimiento**

Pedro Nel Gómez Moreno

Fecha de recepción: 5-09-18

* MSc en Psicología de la Educación, Especialista en Necesidades Educativas e Inclusión, Licenciada en Lengua Castellana y Comunicación Social, Docente de básica primaria en la I.E Pozo Nutrias Dos de San Vicente de Chucuri Santander lucecilla621@gmail.com

* MSc en Psicología de la Educación, Especialista en Necesidades Educativas e Inclusión. Psicóloga, Docente Orientadora en la

I. E. Pozo Nutrias Dos de San Vicente de Chucuri Santander,

\section{PARA CITAR ESTE ARTÍCULO}

Duarte, L., Pabón, N., Ascencio, A. y Gómez, P. (2019).Discapacidad visual. Una experiencia en el profesorado estatal del Departamento de Santander, Colombia. Nodos y nudos, 6(46), 13-26. doi: 10.17227/nyn.vol6. num46-10597

$* *$ en Necesidades Educativas e Inclusión. Psicóloga, Docente
Orientadora en la I. E. Colegio Integrado Nuestra Señora de la Paz Betulia Santander. angieascencio@hotmail.com

MSc. Psicología Clínica Infantojuvenil (Instituto Superior de Estudios Pscicológicos, isep, Barcelona, España). Licenciado en Educación Básica con énfasis en Humanidades y Lengua Castellana (U. De Pamplona). Especialista en Educación con Nuevas Tecnologías ( Universidad Autónoma de Bucaramanga, unab). Docente catedrático en la Especialización en Necesidades Educativas e Inclusión y evaluador de proyectos de grado de la Maestría en Educación. 


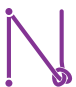

Volumen 6 N. ${ }^{\circ} 46$ enero - junio de 2019 ISSN: 0122-4328 ISSN-E: 2619-6069 pp. 73-88

\section{RESUMEN}

El artículo de investigación surge como parte del proceso de la Maestría en Psicología de la Educación cuyo propósito fue comprender el significado de la experiencia docente del profesorado estatal de discapacidad visual del Departamento de Santander, Colombia. Lo anterior se realizó a través de una metodología cualitativa, basada en un paradigma fenomenológico y el estudio de caso. Como resultado se obtuvo que las historias de vida son complejas y cargadas de sufrimiento tanto a nivel personal como familiar y social; son además marcadas por la pobreza y la violencia, por lo que su discapacidad visual atravesó procesos críticos; sin embargo, las aptitudes adquiridas durante la formación personal y profesional fueron un gran logro para las personas que formaron parte del estudio. Se concluyó que el significado de la experiencia en el profesorado estatal del Departamento de Santander fue muy importante; esta contribuyó a reconocerse como profesionales capaces de construir sociedad. Así mismo, la experiencia como docentes les permitió entender que ver va más allá del sentido de la visión, es sentir en sus estudiantes al "ser" individual que piensa y expresa sus necesidades; lo más importante es la actitud, lo que hace que esta experiencia sea trascendental.

Palabras clave: discapacidad visual; experiencia docente; educación inclusiva

\section{Abstract}

This research article emerges as part of the process of the Master in Educational Psychology whose purpose was to understand the meaning of the teaching experience of the state teachers with visual impairment of the Department of Santander, Colombia. This was done through a qualitative methodology, based on a phenomenological paradigm and a case study. As a result, it was obtained that life stories are complex and loaded with suffering at the personal, family, and social level. They are also marked by poverty and violence, so that their visual impairment went through critical processes. However, the skills acquired during personal and professional training were a great achievement for the people who were part of the study. It was concluded that the meaning of the experience in the state teachers of the Department of Santander was very important. This helped recognize themselves as professionals capable of building society. Likewise, their experience as teachers allowed them to understand that seeing goes beyond the sense of vision, it is to feel in their students the individual "being" that feels, thinks and expresses his needs. The most important thing is the attitude, which makes this experience transcendental.

Keywords: visual impairment; teaching experience; inclusive education

\section{Resumo}

0 artigo de pesquisa surge como parte do processo do Mestrado em Psicologia Educacional, cujo objetivo era compreender o significado da experiência de ensino do corpo docente estadual com deficiência visual do Departamento de Santander, Colômbia. Isso foi feito através de uma metodologia qualitativa, baseada em um paradigma fenomenológico e no estudo de caso. Como resultado, obteve-se que as histórias de vida são complexas e carregadas de sofrimento, tanto pessoal quanto familiar e social; elas também são marcados pela pobreza e pela violência, de modo que sua deficiência visual passou por processos críticos. No entanto, as habilidades adquiridas durante o treinamento pessoal e profissional foram uma grande conquista para as pessoas que fizeram parte do estudo. Concluiu-se que o significado da experiência no corpo docente estadual do Departamento de Santander era muito importante; ista ajudou a se reconhecer como profissionais capazes de construir a sociedade. Da mesma forma, a experiência como professores Ihes permitiu entender que a visão vai além do sentido da visão, é sentir em seus alunos o "ser" individual que sente, pensa e expressa suas necessidades. 0 mais importante é a atitude, que torna essa experiência transcendental.

Palavras-chave: deficiência visual; experiência docente; educação inclusiva 


\section{Introducción}

Este artículo es producto de la investigación que se desarrolló en el Departamento de Santander con docentes de aula y de apoyo de discapacidad visual vinculados al servicio estatal, que laboran como docentes en Instituciones Educativas de carácter rural y urbano.

El objetivo es comprender los significados construidos sobre sus experiencias docentes, en el marco de las oportunidades de acceso y permanencia en el sistema de educación formal, visibilizando así posibles procesos de inclusión o exclusión.

El Ministerio y las Secretarias de Educación cuentan con lineamientos para una educación inclusiva como estrategia de inclusión social e implementación de ambientes de este tipo en educación superior. Algunos de estos lineamientos hacen referencia a la gestión territorial para el incremento de la cobertura, la aplicación de la evaluación de los estudiantes con discapacidad para ser bachilleres, a la infraestructura, a la educación especializada elaborada, a la inclusión de niñas, niños y adolescentes con necesidades especiales asociadas a la discapacidad, a la entrega de material para mejorar la calidad de la educación, entre otros aspectos (Gutiérrez, 2018). Sin embargo, es relevante analizar desde la voz de los actores sociales aquellas situaciones que viven cotidianamente en el ejercicio de su profesión, por lo que resulta pertinente reconocer la historia de su vinculación a la carrera docente después de haber culminado sus estudios profesionales e identificar las formas en que han asumido el trabajo y desempeñado sus roles.

Se espera que este estudio brinde elementos de reflexión para las políticas públicas relacionadas con la inclusión social y laboral, dado que se considera que estos procesos deben ser jalonados desde diferentes sectores sociales, permitiendo así progresos significativos en la promoción de los derechos de la población con discapacidad y en la garantía y fortalecimiento de su dignidad, asuntos que implican el otorgamiento de oportunidades equitativas.

En consonancia con lo anterior, la ley General de Educación establece que "Las personas con limitaciones y con capacidades o talentos excepcionales son parte integrante del servicio público educativo" (Ministerio de educación, 2018 p. 12). Sin embargo, la realidad normativa no siempre está articulada con la realidad social, por lo que es relevante comprender qué ha significado y qué significa para los docentes ejercer su profesión en diferentes Instituciones Educativas de Santander.

Los procesos de inclusión que se desarrollan en sociedades modernas surgen de las luchas de grupos de la sociedad civil que incitan a los entes oficiales a proteger los derechos de las personas con discapacidad para que logren un desarrollo integral y una mejor calidad de vida.

La inclusión social y educativa ha venido evolucionando y ha ganado un mayor reconocimiento por parte de la legislación colombiana, algo que es relativamente nuevo. Las leyes 762 de 2002, 1145 de 2007 y la ley estatutaria 1618 de 2013 han venido gestándose a nivel nacional, promoviendo los medios para que los niños, niñas y adolescentes sean aceptados, integrados y sostenidos en el sistema educativo con el fin de favorecer la formación integral equitativa según sus diferentes capacidades.

En la situación particular de la discapacidad visual se han desarrollado oportunidades en las universidades que ofertan carreras en ciencias humanas, sociales y arte, tales como: Licenciaturas, Trabajo Social, Sociología, Psicología, Derecho, Artes, Música y otras; para ello, se han adaptado los recursos como el software especializado y los dispositivos que codifican el Braille y facilitan el desarrollo de los trabajo y los exámenes a los estudiantes que presentan dicha discapacidad (Cano, 2016). Con respecto a la carrera docente se ha observado un avance en torno a la aceptación y a las oportunidades de ejercer el quehacer pedagógico que recoge el espíritu de las normas que buscan garantizar la oportunidad de derechos a todos los ciudadanos de acuerdo con la Constitución Política de Colombia de 1991. Un ejemplo de ello son los hermanos Olascoagas, docentes con discapacidad visual que se desempeñan en una Institución educativa de la ciudad de Medellín. Además de ejercer su quehacer pedagógico, ellos han brindado la oportunidad a otras personas en situación de discapacidad visual para la superación personal y profesional.

Con la aprobación de la ley 97, que obedece al cierre de las escuelas para personas con discapacidad, los hermanos deciden crear Corpanin, fundación que 
se encarga de potenciar las dimensiones humanas de los niños y jóvenes con discapacidad visual. Esto lo logran a través de programas con estimulación infantil adecuada, el desarrollo de habilidades pedagógicas y socio-ocupacionales, además de asesorías y talleres artísticos y vocacionales (Kienyke, 2016, p. 1).

Según el Decreto 1278 de junio 19 de 2002, por el cual,

Se expide el Estatuto de Profesionalización Docente en Colombia, el único requisito para que una persona pueda ejercer la carrera docente es ser Normalista Superior para Preescolar y Primaria, o tener pregrado en Licenciatura en Educación, en cualquiera de las áreas académicas o profesionales. (Ministerio de Educación, 2002).

Un educador con discapacidad visual en Colombia tiene menos oportunidades de acceder a la carrera docente puesto que debe participar como todos en un concurso de méritos que, aunque se le facilita personal de asistencia, como lectores que le prestan el servicio durante el examen de competencias básicas, es complicado para ellos hacer imagen mental de los ejercicios de matemáticas que tienen figuras, barras, formulas, etc. Hace falta que las pruebas sean más descriptivas y más adecuadas a todo tipo de persona, de manera que se den las mismas oportunidades a todos en igualdad de condiciones (Gómez, 2017). Esta misma dificultad se presenta para acceder a nombramientos provisionales o reemplazos de cualquier índole por las barreras que impiden la posibilidad de ser considerados "aptos" (Gómez, 2017).

No obstante, es necesario reconocer el esfuerzo de algunos programas que buscan precisamente romper esas limitaciones, como el del "Banco de la Excelencia", plataforma virtual del Ministerio de Educación que a partir del año 2016 se convirtió en un instrumento para otorgar nombramientos provisionales o reemplazos según el perfil, la experiencia y demás cualidades requeridas para esos cargos. (MEN, 2016b)

Los accesos a estas oportunidades laborales son limitados aún más en un contexto como el colombiano que tiene altos índices de desempleo. Se considera así que el pais debe sumar esfuerzos para promover la cultura de la inclusión laboral de docentes con discapacidad dado que es indispensable para el desarrollo armónico de una nación y el reconocimiento de la igualdad de oportunidades basadas en los méritos y en las cualidades de las personas, no en sus condiciones físicas. De acuerdo con el informe de RCN (2017), la Universidad La Gran Colombia realizó un estudio sobre el desempleo de personas en situación de discapacidad visual; se conoció que la tasa de desempleo es siete veces más que la del pais, es decir, el 62\%, correspondiendo el $69 \%$ a mujeres y el 54\% a hombres.

\section{La educación inclusiva}

Según Arizabaleta y Ochoa (2016), la educación inclusiva en Colombia es la gestión de la transformación y la adaptación de las instituciones educativas a los distintos obstáculos de sus participantes para diseñar procesos de aprendizaje inclusivos que permitan la formación tanto de la comunidad estudiantil como de los maestros; en un proceso que pase de la homogeneidad en la academia y se adopte la heterogeneidad, promoviendo la participación de los involucrados como sujetos de derechos.

La educación inclusiva es un enfoque que propone la eliminación de las barreras, estigmas y métodos excluyentes para que todos los estudiantes tengan oportunidades de ingreso, atención, permanencia, evolución y sostenibilidad dentro del sistema educativo $y$, posteriormente, proyección en la vida social, teniendo en cuenta sus necesidades especificas. Esta acerca a la población a una educación equitativa y de calidad para todos (Arizabaleta y Ochoa, 2016).

La educación inclusiva implica que los niños, niñas y jóvenes de una determinada comunidad aprendan juntos independientemente de sus necesidades o situaciones especificas, valorando más sus habilidades que sus dificultades en el proceso de enseñanza-aprendizaje (Arizabaleta y Ochoa, 2016).

Se toma como referente de origen de la Educación Inclusiva la "Declaración de Salamanca" (1994), que recomienda ver con normalidad las diferencias y dar espacios para el desarrollo de atención a la diversidad en aulas regulares, teniendo la capacidad de educar desde un enfoque diferencial a cada uno de los estudiantes (Unesco, 1994).

Por otra parte, Ainscow (2001) señala que la educación inclusiva: 
Es un proceso de incremento de la participación de los alumnos en las culturas, currículos y comunidades de sus escuelas locales y de reducción de la exclusión de los mismos, sin olvidar, por supuesto, que la educación abarca muchos procesos que se desarrollan fuera de las escuelas. (p. 42).

La Unesco (2006) define así mismo la educación inclusiva como:

El proceso de abordar y responder a la diversidad de necesidades de todos los alumnos a través de prácticas inclusivas en el aprendizaje, las culturas y las comunidades; y reducir la exclusión dentro de la educación implica cambios y modificaciones en el contenido, los enfoques, las estructuras y las estrategias, con una visión común que cubra a todos los niños del rango apropiado de edad y una convicción que es responsabilidad del sistema ordinario, educar a todos los niños (p. 22).

Del mismo modo, el currículo inclusivo que hace referencia a las adaptaciones que se generan para la atención de los estudiantes con alguna discapacidad o situación particular es visto como la reflexión permanente para lograr una educación más significativa, teniendo en cuenta las características del contexto y generando propuestas de esquemas curriculares reales enfocados desde el P.E.I. (Proyecto Educativo Institucional) (Arizabaleta y Ochoa, 2016).

El currículo flexible debe contemplar un contenido dinámico que favorezca los diversos ritmos y estilos de aprendizaje, promoviendo la necesidad del estudiante de aprender desde su propio entorno y con sus propias habilidades adaptativas. Teniendo en cuenta que todos los casos son diferentes, los seguimientos y las evaluaciones son individuales y particulares.

\section{El diseño universal del aprendizaje}

El diseño universal de aprendizaje es un método que tiene en cuenta a la diversidad, tanto en las prácticas educativas como en la accesibilidad, el uso arquitectónico, los avisos, el LSC-Braille y los dispositivos tecnológicos. Este busca generar adaptaciones a cualquier tipo de necesidad, limitaciones o barreras, accediendo de forma universal a la información.

Es así como las universidades deben adaptar sus sistemas para que la población con discapacidad visual pueda acceder a la educación al igual que los otros estudiantes, y, de la misma manera, para que los docentes con las mismas dificultades puedan contar con dichas herramientas que son fundamentales para el diseño y la aplicación de una enseñanza efectiva. Según Cast (2008), "para alcanzar altos niveles de rendimiento en el proceso enseñanza-aprendizaje, se debe rechazar el principio de que una misma talla vale para todos" (p. 11). Por el contrario, se debe implementar un diseño flexible con opciones adaptables a las necesidades individuales y con ello optimizar la enseñanza para los diversos aprendices que se encuentran en las aulas de clase.

Por otra parte, Galvis (2007) define el perfil docente como "[...] el conjunto de competencias organizadas por unidades de competencias, requeridas para realizar una actividad profesional, de acuerdo con criterios valorativos y parámetros de calidad" (p. 18). Estas competencias abarcan la formación en valores, la comprensión del contexto como base de la enseñanza y aprendizaje, la capacidad para relacionarse asertivamente con estudiantes y padres de familia, la idoneidad en su quehacer, el amplio conocimiento respecto a lo que enseña, la habilidad para planear y organizar, la facilidad para involucrar a los estudiantes en el proceso de enseñanza e interactuar en la convivencia escolar. Por tanto, proyecta al estudiante como artífice de su propio aprendizaje, generando expectativas en cuanto a su proyecto de vida y a su inclusión en ámbitos laborales y sociales, desarrollando habilidades éticas, criticas, creativas y productivas.

Estas acciones son las que debe tener en cuenta el docente en la planeación y ejecución de sus orientaciones escolares. De este modo, se ratifica como "la capacidad del individuo para el cumplimiento de sus funciones, acciones y papeles propios de la actividad pedagógica profesional" (Roca, citado por el Ministerio de Educación, 2016a, p. 8).

Las competencias del docente son el compendio de habilidades encaminadas al desarrollo de acciones pedagógicas que buscan la formación integral de los estudiantes. Sus ejes transversales para el logro de dicha integralidad son el Ser, el Saber y el Convivir. Tomando como referencia el planteamiento señalado por Delors (1996), en el informe presentado a la Unesco 
con la Comisión Internacional sobre Educación para el siglo XXI, la educación tiene cuatro pilares fundamentales para el aprendizaje: (1) saber ser, (2) saber aprender, (3) saber hacer docencia y (4) saber convivir.

Fernández, (2013) afirma que,

En esta línea han sido muchos los trabajos que se han realizado con relación a las competencias docentes, aunque todavía desconocemos que capacidades o competencias específicas relacionadas con la atención a la diversidad puedan ser utilizadas como herramienta poderosa para favorecer la inclusión. (p. 19).

\section{La discapacidad visual}

La persona con discapacidad visual o invidencia total es quien tiene un impedimento general de proyección a través de la retina, imposibilitando realizar tareas o ejecuciones visuales; cabe aclarar que existen varios tipos de discapacidad: visual, adquirida y congénita, es decir, en algunos casos las personas nacen con su visión normal y debido a situaciones como accidentes, cirugías, traumas celébrales y demás, pueden perder la visión, caso contrario a la persona que nace siendo ciega.

Según el libro Soluciones para la baja Visión, Arroyo (2013) presenta las siguientes definiciones de acuerdo con la complejidad de la agudeza visual:

»Ceguera: Carencia de visión o solo percepción de luz, imposibilidad de realizar tareas visuales.

» Discapacidad visual profunda: dificultad para realizar tareas visuales gruesas, imposibilidad de realizar tareas que requieran visión de detalles.

» Discapacidad visual severa: posibilidad de realizar tareas con errores, con requerimientos de adecuación de tiempo, ayudas y modificaciones.

" Discapacidad visual moderada: posibilidad de realizar tareas visuales con el empleo de ayudas especiales e iluminación adecuada, similares a las que realizan personas con visión normal (p. 57).

La discapacidad visual en la actualidad ya no es un impedimento para que tanto estudiantes como maestros interactúen en el proceso de enseñanza-aprendizaje, por el contrario, con la inclusión se logra abrir muchas puertas para que los estudiantes en situación de discapacidad puedan asistir al aula, optando por utilizar el lenguaje Braille y otros dispositivos electrónicos como ayuda en dicho proceso. Así mismo, los maestros en situación de discapacidad pueden apoyarse en este tipo de herramientas para impartir sus clases de una manera óptima para mejorar el aprendizaje y adquisición de conocimientos de los estudiantes.

Finalmente, es importante resaltar el índice de inclusión que es una guía que permite visualizar cómo se encuentran realmente las escuelas en las estrategias de atención a la diversidad. Este es un proceso de análisis o autoevaluación contextualizada que identifica mejor en qué aspectos deben mejorar la prestación del servicio educativo, teniendo en cuenta las diferentes necesidades de los estudiantes. El proceso radica en la implementación de dos cuestionarios que involucran la participación de docentes, directivos docentes, administrativos, personal de apoyo, padres de familia y estudiantes. Se evalúa por medio de parámetros que van con los calificativos de "siempre, casi siempre, algunas veces, no sé y no se hace".

El Índice se va construyendo sobre la base del conocimiento de todos los miembros de la comunidad educativa y se adapta a las circunstancias específicas de esta. De este modo, anima a todo el centro a sentirse partícipe y protagonista del proceso de desarrollo de una escuela inclusiva (Ainscow, 2001, p. 8).

El índice de inclusión debe servir para mejorar el Proyecto Educativo Institucional. Este es un documento que, de acuerdo al contexto, contiene las normas constitucionales y lineamientos del Ministerio de Educación Nacional. Su objetivo es direccionar el horizonte institucional, los planes de área, el currículo, el manual de convivencia, el manual de funciones administrativas, los proyectos transversales y el sistema de evaluación institucional pertinentes de cada institución en particular. Es evaluable y ajustable anualmente mediante planes de mejoramiento institucional, de modo que se redireccionan los logros tendientes a alcanzar. Así, tanto el índice de inclusión como el pel son herramientas que ayudan a fortalecer la Educación Inclusiva en contextos locales, regionales o nacionales. 
Según Colombia aprende (2005),

El pel es la carta de navegación de las escuelas y colegios, en donde se especifican, entre otros aspectos, los principios y fines del establecimiento, los recursos docentes y didácticos disponibles y necesarios, la estrategia pedagógica, el reglamento para docentes y estudiantes y el sistema de gestión. El proyecto educativo institucional debe responder a situaciones y necesidades de los educandos, de la comunidad local, de la región y del pais, ser concreto, factible y evaluable (párr. 1).

\section{Diseño metodológico}

Un diseño metodológico cualitativo, según Sandoval (1996), se entiende como el proceso de caracterización de un fenómeno por medio de sus cualidades. No obstante, su significado es mucho más amplio ya que corresponde a un abanico diverso de formas de comprender los significados y sentidos a partir de los cuales se configura la realidad.

Se asume, desde este ángulo, que la construcción de conocimiento es una co-creación fundada en la interacción entre el investigador y el investigado; de allí que los procesos subjetivos e intersubjetivos actúen como puentes facilitadores para comprender las realidades humanas en sus contextos particulares. Desde este enfoque, la realidad social aparece fragmentada, no siendo posible abordarla a partir de criterios de objetividad; hay pues una heterogeneidad propia de los fenómenos sociales, una multiplicidad de singularidades desde los cuales pueden ser enunciados.

El ser humano históricamente tiende a buscar soluciones a las distintas problemáticas e incertidumbres que emergen de la cotidianidad y es una condición de su ser social, tanto de forma empírica como usando formas y métodos que en ocasiones son inconscientes. Es una búsqueda continua de respuestas a su propia existencia; sin embargo, aspectos como la moralidad, la libertad y las acciones humanas son de dificil comprensión ya que dependen de fenómenos subjetivos y relativos que pueden ser interpretados de formas disímiles.

Partiendo de lo anterior, se asume una postura metodológica de carácter fenomenológico, la cual no parte del diseño de una teoría, sino que permite relacionarse a profundidad con el mundo del actor social, realizando un análisis descriptivo de su experiencia, sin acudir a explicaciones causales.

\section{Población y muestra}

Los participantes fueron docentes con discapacidad visual, específicamente cinco (5) profesores que laboran como docentes de apoyo y de aula regular (dos en área rural y tres en zona urbana) en instituciones públicas del departamento de Santander; sus edades oscilan entre 40 y 55 años. Estos casos fueron seleccionados por encontrarse en el área metropolitana de Bucaramanga (Santander), facilitando el proceso de generación de información.

\section{Instrumento}

Se realizaron entrevistas a profundidad. En esta técnica el entrevistador guía el diálogo y permite que el entrevistado exponga sus puntos de vista. Es una entrevista abierta enfocada hacia "la comprensión de las perspectivas que tienen los informantes respecto de sus vidas, experiencias o situaciones, tal como las expresan con sus propias palabras" (Taylor y Bogdan, 1987, p. 47).

Las entrevistas fueron grabadas en audio y posteriormente transcritas sin cambiar ni modificar los datos de información. Posteriormente, se utilizó el software Atlas TI para el análisis de la información.

\section{Resultados. Análisis de categorías de la discapacidad}

La discapacidad hace referencia a un concepto global que reúne un conjunto de deficiencias del ser humano en las funciones corporales y en las limitaciones de la capacidad para realizar actividades y participar socialmente (Hernández, 2015).

La discapacidad (visual, auditiva, sensorial, cognitiva, psíquica, entre otras) afecta el nivel de vida de quienes las padecen, sin embargo, depende de ellas mismas la superación y el esfuerzo para lograr sus metas.

Según Hernández (2015), "En Colombia se ha dado un gran desarrollo normativo en favor de las personas con discapacidad, sin embargo, es a partir de la Constitución Política de 1991 que la discapacidad es entendida en términos de derechos" (p. 55). 
Lo anterior demuestra que el Estado, como garante de la equidad, la inclusión y los derechos de las personas, está velando por llevar a cabo acciones asertivas en favor de poblaciones vulnerables, promoviendo políticas de previsión, rehabilitación e integración social, pero, sobre todo, de inclusión social, laboral, cultural y educativa.

Para el caso de los docentes que formaron parte del estudio, se evidencia que todos han buscado en la familia, la escuela, la sociedad y sobre todo en el Estado apoyo para continuar adelante con sus proyectos de vida.

\section{Significado de las experiencias}

Las experiencias de los docentes han sido relevantes dado que han permitido contribuir a la formación de las personas por medio de la pedagogía impartida sin importar la discapacidad visual y las limitaciones que esta genera.

El significado de las experiencias está dado tanto por la orientación de estrategias pedagógicas como por el trabajo en conjunto con los pares. Así, se construye sociedad a partir de la enseñanza sin que la discapacidad limite las competencias personales y laborales.

\section{Retos personales}

Los retos personales de los docentes que formaron parte del estudio son admirables, no solo por exponerse a un mundo donde existen limitaciones para estas personas, sino porque las oportunidades laborales para las mismas son reducidas.

Se puede decir que la inclusión ha ganado relevancia en los distintos escenarios (educativos, deportivos, culturales y sociales); sin embargo, de acuerdo con el Ministerio de educación, cultura y deporte (2012), el fenómeno de la exclusión social en la última década está adoptando dimensiones cada vez más preocupantes ya que son los propios sistemas educativos los que se convierten en la primera fuente de promoción aunque no sea tan visible.

Uno de los retos personales de los docentes ha sido aprender el lenguaje Braille y el uso de dispositivos electrónicos que ayudan en el proceso de aprendizaje personal.
Otros retos más sobresalientes que afrontaron fueron:

» Adaptarse a la actitud de sus maestros.

» Tener acceso a las oportunidades laborales.

» Lograr títulos académicos.

» La presentación de los exámenes de evaluaciones y admisiones.

"El contacto con los estudiantes y docentes sin discapacidad.

» Aprender a conocerse en su capacidad de superar dificultades.

»El aprendizaje de otros idiomas.

\section{Afrontamientos personales}

Los afrontamientos personales de cada uno de los docentes en estudio son admirables porque han tenido que luchar consigo mismos y afrontar la situación desde la realidad. Porque siempre mantuvieron una actitud fuerte frente a la vida, unas ganas persistentes de superación y no se dejaron vencer ante ningún obstáculo.

Todos han luchado desde que se dieron por enterados de su discapacidad por desarrollar competencias y facultades para contribuir a la sociedad en el campo de la pedagogía sin que su limitación física se los impidiera; todos han adoptado aptitudes positivas frente a la vida, lo que ha conllevado a lograr mayores aprendizajes, eliminar barreras mentales y ser útiles a la sociedad.

Los mayores afrontamientos personales fueron sentirse capaz y aceptar su discapacidad y, en segundo lugar, tener la suficiente gallardía para afrontar las dificultades y lograr los objetivos propuestos: aprender y alcanzar su profesionalización para ser alguien útil e importante para el mundo.

\section{Prácticas pedagógicas - Rol del docente}

Las prácticas pedagógicas de los docentes con discapacidad visual no han sido fáciles; por el contrario, ha sido un reto personal y profesional para adquirir el 
conocimiento, y poder así brindar orientación a través del proceso de enseñanza-aprendizaje.

Según Ramírez (2016), en las prácticas pedagógicas, "los docentes buscan inspirar y motivar a los estudiantes con la implementación de diferentes lenguajes (verbal, icónico, entre otros) con el fin de alcanzar y lograr los propósitos de aprendizaje" (p. 118).

Las actividades, las estrategias y las acciones pedagógicas se ejecutan y diseñan sobre la planeación del proceso de enseñanza y aprendizaje, dependiendo de las necesidades de los estudiantes. Con este objetivo, se adecúan las formas de presentar los contenidos, se incluyen herramientas de las TIC, se realizan talleres de sensibilización y se realiza la planeación de los objetivos anuales, la comunicación y la promoción del trabajo en equipo.

\section{Educación inclusiva}

La inclusión es un concepto que día a día está siendo tenido en cuenta por personas e instituciones en los cuales se valoran los derechos que tienen todas las personas independientemente de su situación de discapacidad. La inclusión es importante entenderla desde sus distintas dimensiones: social, cultural, pero sobre todo educativa.

La inclusión social dentro de los discursos del Derecho y su reconocimiento por las legislaciones en Colombia constituye algo relativamente nuevo. Las leyes 762 de 2002, 1145 de

2007 y la ley estatutaria 1618 de 2013 han venido gestándose a nivel nacional, promoviendo los medios para que las personas en situación de discapacidad sean aceptados, integrados y sostenidos en el sistema educativo para favorecer la formación integral equitativa.

Las dimensiones inclusivas del derecho a la educación se ven reflejadas en la enseñanza a todas las personas con discapacidad física, cognitiva, mental o psíquica, sin importar su género o raza. La constitución estipula además que dicha enseñanza ha de ser accesible a los minusválidos y a los grupos menos favorecidos desde el punto de vista social y económico, como los inmigrantes, los refugiados y los miembros de minorias (comprendidos los pueblos indígenas), los jóvenes desfavorecidos y marginados, a fin de que se integren más fácilmente a la sociedad.
Según Vásquez (2012), "la educación inclusiva se apoya en la convicción de que todos los alumnos pueden aprender cuando se les otorgan las oportunidades de aprendizaje apropiadas" (p. 5).

Es importante resaltar que cuando se planea el aprendizaje individualizado, se crean equipos de apoyo, se estimulan las responsabilidades sociales entre el alumnado, se trabaja en colaboración con los padres además con los otros miembros de la comunidad y se aplican planes de formación del personal. Se puede decir que en las instituciones se han adoptado los principios de la educación inclusiva (Vásquez, 2012, p. 5). Para el caso de los docentes que formaron parte de este estudio, la educación inclusiva ha permitido la eliminación de barreras y el ingreso a instituciones educativas sin estigmas ni métodos excluyentes; ellos han tenido la oportunidad de compartir con otros, trabajar en equipo por el mismo ideal: enseñar y realizar prácticas académicas que contribuyan a formar una sociedad mejor y unos profesionales idóneo para esta misma.

\section{Análisis de los resultados}

Como se mencionó anteriormente, se realizó una entrevista a profundidad con la finalidad de visibilizar el significado de la experiencia docente del profesorado estatal con discapacidad visual en el departamento de Santander. Se analizaron las historias de vida de cuatro docentes adscritos a las SED de Santander y de Bucaramanga; así mismo, se identificaron los retos y afrontamientos personales que determinaron actitudes y aptitudes que incidieron favorablemente en la educación inclusiva en Santander.

En lo que respecta a la presentación de los resultados e interpretación de las entrevistas, se hizo un análisis desde la perspectiva de distintas subcategorías codificadas como se presentan a continuación.

Al adentrarse en lo que dicen los docentes de la categoría de historias de vida, se obtuvo lo siguiente: se pudo percibir que las historias de vida son complejas y muy difíciles, cargadas de sufrimiento tanto a nivel personal como familiar y social; están marcadas por la pobreza y la violencia, de modo que su discapacidad atravesó procesos críticos llenos de lucha por la incomprensión de los padres ante la problemática y su incapacidad para solucionarla, y por la 
búsqueda constante de un tratamiento médico para obtener lo que se ya había perdido: la visión.

La discapacidad de las personas que formaron parte del estudio fue adquirida en distintas etapas de su vida: para cuatro de ellos en la infancia por enfermedad, accidente, infección y para uno en la adultez temprana debido a un error médico. Todos sintieron sus sueños truncados pero buscaron la forma de salir adelante, de continuar con sus estudios pese a las injusticias de algunos docentes que no comprendian en totalidad la discapacidad presentada.

\section{La orientación sobre estrategias pedagógicas}

El significado de las experiencias ha sido relevante y a su vez contradictorio porque a pesar de que iniciaron tardiamente la primaria por falta de rehabilitación, dificultades en la salud y por no contar con apoyo especializado, además de que algunos vivían en espacios rurales, los docentes han desarrollado una gran cantidad de habilidades que antes no creían tener; una de ellas fue aceptar su dicapacidad pese a la reacción y comportamiento de los demás (profesiones, compañeros y padres) y otra la obtención de conocimiento del lenguaje o sistema en Braille, el cual les permitió adquirir un conocimiento especializado y brindarlo desde la docencia a muchos estudiantes que lo necesitan. Así mismo, la experiencia como docente les permitió entender que ver va más allá del sentido de la visión, es sentir en sus estudiantes al "ser" individual que piensa y vive una experiencia única; que es necesario entender al otro, ponerse en su lugar, conocerlo, saber escucharlo, y que finalmente lo más importante es la actitud, lo que hace de esta experiencia una experiencia trascendental.

Al adentrarse en lo que dicen los docentes de la categoría de identificación de los retos y afrontamientos personales, se obtuvo manifestaciones como las que siguen:

El reto fue inicialmente con ellos mismos al tener que pensar en cambiar de actitud, la parte actitudinal, al ser discriminados, llamados por algunos "incapaces"; entonces la meta era demostrarse a ellos mismos y a los demás hasta donde se podía llegar.
Los mayores afrontamientos personales fue sentirse capaz y aceptar, en primer lugar, su discapacidad $y_{1}$ en segundo lugar, tener la suficiente gallardia para afrontar las dificultades y lograr los objetivos propuestos, aprender y alcanzar su profesionalización para ser alguien útil e importante para el mundo.

Es así como logran adquirir competencias para ejercer la docencia con dificultades, pero con la gran satisfacción de ser útil y tener la oportunidad de brindarle a otro las herramientas necesarias para desarrollar sus propias habilidades y capacidades en un ambiente agradable, brindando conocimientos en una constante formación.

Los aportes que hacen son importantes para los procesos de educación inclusiva, su experiencia de vida, sensibilidad, fortaleza, practicas inclusivas, creatividad, desarrollo de competencias, apoyo a sus pares académicos, padres de familia y a sus estudiantes sirvieron de base para la adecuación de los Proyectos Educativos Institucionales (P. E. ı.), desarrollando los currículos en favor de la población estudiantil existente y garantizando el acceso, sostenibilidad y promoción de las futuras generaciones.

Al adentrarse en lo que dicen los docentes de la categoría actitudes, se encuentra que las aptitudes adquiridas durante el arduo trabajo de la formación personal y profesional son un gran logro. Ellos no se conformaron con el pregrado, han realizado especializaciones y maestrías, en su constante motivación por superarse día a día. Las actitudes percibidas en los entrevistados son muy positivas ya que ven en la discapacidad otra forma de desarrollar habilidades y destrezas para su vida personal como profesional y laboral, sirviendo de referentes de superación a toda la comunidad educativa y siendo modelos para otras personas con la misma discapacidad.

Por otra parte, se considera que la educación inclusiva permite la eliminación de barreras y, a su vez, permite la interacción con otros, la realización de trabajo en equipo, la práctica de una escuela nueva, el ejercicio de valores permanentes y, con ello, las oportunidades de evolución y sostenibilidad dentro de un sistema educativo con mayor proyección en la vida social. 
Cabe resalar que para los docentes con discapacidad visual fue de gran importancia indagar sobre las experiencias individuales que desarrollan los docentes con esta discapacidad a fin de seguir contribuyendo con los procesos de educación inclusiva y establecer referentes que sirvan de apoyo para nuevas investigaciones y oportunidades a nuevos profesionales.

\section{Discusiones}

Con relación a las categorías de análisis, pudimos observar que fueron enmarcadas por situaciones particulares de la vida de los docentes. En la categoría "Historias de vida" hallamos que no solo la dificultad la daba la discapacidad, sino que cada uno contó con experiencias no gratas a nivel familiar, de discriminación y falta de apoyo, pues la mayoría residia en zonas rurales donde las oportunidades era escases 0 no existían en absoluto. Estas situaciones dificultaron aún más las oportunidades de acceso, atención temprana y desarrollo de habilidades de rehabilitación en base a sus otros sentidos (Hernández, 2015).

Desde la categoría de identificación de los retos y afrontamientos personales, encontramos que las situaciones de sufrimiento lograron forjar sus caracteres dando más impulso al deseo de alcanzar metas. Pero era imposible lograrlo solos, necesariamente tuvieron que contar con el apoyo de personas (familiares, allegados o desconocidos altamente sensibles) que creyeron en ellos, en cómo los veían afrontando su discapacidad con tenacidad, trabajando en cada dificultad presentada.

En este punto resaltamos la importancia del vínculo social, el cual, según Paugam (2012, citado por Jiménez, 2017) atiende tanto una dimensión de protección, como una de reconocimiento, si se mira desde una perspectiva sociológica:

La protección remite al conjunto de soportes que brindan al sujeto la familia, la comunidad, para enfrentar las cambiantes condiciones de la vida. El reconocimiento se entiende como dimensión que parte de la interacción con los otros y que le posibilita al sujeto ver su valía en el grupo, asi se forma parte de un nosotros, que genera interdependencia afectiva. (Jiménez, 2017, p. 81).

El vínculo afectivo es el primer apoyo que reciben las personas con discapacidad, para dar los primeros pasos de adaptación en las instituciones educativas. Los familiares, amigos y profesionales sensibles son quienes, en épocas de poca atención a la defensa de sus derechos, ofrecieron la ayuda necesaria para el acceso, el sostenimiento y promoción en sus diferentes etapas escolares.

Un gran hallazgo encontrado en esta investigación fue descubrir que no basta con generar sensibilidad frente a la situación de discapacidad, definitivamente las personas deben demostrar actitud luchadora, mostrar al que "ve" que la visión no es solo física sino de proyección; a esto se debe que no todos los invidentes logren sus proyectos de vida, son pocos los que lo logran y no solo por falta de apoyo de las instituciones gubernamentales, definitivamente es por la tenacidad, la actitud frente a la vida y al desarrollo de habilidades específicas y particulares. Estos retos y afrontamientos en cada nivel de preparación ofrecieron pruebas de fortalecimiento personal, en cada una el invidente desarrolló y reconoció habilidades y pre-saberes que le orientaron profesionalmente, por consiguiente, la docencia se convirtió en un rol especial, les ofreció y lo sigue haciendo, la concepción de la enseñanza desde su propia vida y en todos sus ámbitos. Incluirse y abrirse paso en el sistema educativo ha sido importante para ellos por la influencia que ejercen en sus estudiantes y pares, basándose en las premisas "Si yo pude, ustedes también", "No hay obstáculos invencibles", "La discapacidad es mental".

En cuanto a la categoría de actitudes y aptitudes, se pudo constatar que la perseverancia y la tenacidad fortaleció su personalidad, sin embargo, fueron las habilidades desarrolladas las que hacen la diferencia en las competencias laborales; deben ser incluso mejores que las personas del común, así lo demostraron estos cinco docente al pasar el concurso de méritos del Magisterio. Además de las habilidades, sus acciones deben ir en ascenso, en un mejoramiento continuo, la actitud que han mostrado frente a sus roles les han dado la experticia para mantener la credibilidad de las personas que les rodean. Cuatro de los docentes se encuentran satisfechos con sus cargos y una está en búsqueda de la pensión por afectaciones de salud. 
Las prácticas pedagógicas de los docentes además de ser variadas y creativas son inclusivas, con el propósito de llegar a toda la población estudiantil y, reciprocamente, los estudiantes hacen lo mismo para hacerse entender de sus maestros (Arizabaleta y Ochoa, 2016). Adicional a estas formas de enseñar está el mensaje inmerso en esta interacción en un ámbito escolar, que es la sensibilidad y la tolerancia ante la diversidad y la diferencia: es una enseñanza de vida que por siempre van a practicar (Ainscow, 2001).

Estas experiencias de vida son las que han dado a los docentes el significado en el ejercicio de la docencia.

\section{Conclusiones}

Los docentes en situación de discapacidad visual se describen como personas de carácter fuerte, rígidas, exigentes, de temple, que aprendieron a luchar frente a todas las adversidades, pero sensibles y dispuestas al servicio, humanos y receptivos.

En su historia de vida recalcan episodios de lucha y sufrimiento familiar y personal, mencionan épocas que les dejaron muchas enseñanzas, pero marcadas por huellas de duelo de algunos procesos críticos.

La experiencia en los diferentes ciclos escolares fue contradictoria por la época, iniciaron tardíamente la primaria por falta de rehabilitación, dificultades en la salud y la falta de apoyo especializado, dado que tenian una vivienda rural.

La concepción que tienen de su discapacidad frente a otras es favorable ya que asumen conocer personas con otras discapacidades y destacan la importancia de la comunicación verbal para favorecer la interacción con las demás personas.

En el ingreso a la educación superior se percibe que lograron ingresar con mucha dificultad y con edad de adulto joven. En cuanto a los retos y afrontamientos cada uno, desde su experiencia de vida, los expresa como pruebas difíciles, aunque en cada caso contaron con personas (familiares y amigos) que los apoyaron incondicionalmente hasta conseguir sus metas gracias a su actitud frente a la vida y la fuerza para superar las dificultades. También con la aptitud y su confianza en sus habilidades, cada uno probó de muchas formas que las barreras son mentales y que la concepción de la discapacidad va más allá de los sentidos, es la convicción propia de la voluntad de ser.

Las habilidades adaptativas de estos docentes son un ejemplo de procesos mentales ejemplares porque asumieron en cada situación de vida el reto de enfrentarse a lo desconocido utilizando en su máxima expresión las demás facultades humanas, potencializando sus otros sentidos, la agudeza auditiva, la sensibilidad al tacto, la intuición, la percepción y la memoria. Estos aprendizajes les proporcionaron la experticia necesaria para orientar desde lo humano, lo social y lo académico a los estudiantes que tienen a su cargo, e igualmente a sus pares académicos y padres de familia, apoyándolos en los procesos de educación inclusiva que se da en sus comunidades.

Para ellos el significado de la experiencia es muy profundo y lleno sentido porque les inculcó que para lograr sus metas debian ser los mejores, desarrollando así al máximo sus otros sentidos y teniendo en su vida convicciones claras; por eso ser docentes con discapacidad visual es la profesión desde la cual se construye sociedad, se dignifica a la persona y sus posibilidades, convirtiéndose en un modelo de superación de los obstáculos y las dificultades.

\section{Referencias}

Ainscow M. (2001). Desarrollo de escuelas inclusivas: ideas, propuestas y experiencias para mejorar las instituciones escolares. España: Narcea.

Ainscow, M. y Booth, T. (1995). Índice de inclusión: desarrollando el aprendizaje y la participación en las escuelas. Chile: Unesco, Regional Office for Education in Latin America and the Caribbean, Centre for Studies on Inclusive Education.

Arizabaleta, S. L. y Ochoa, A. F. (2016). Hacia una educación superior inclusiva en Colombia. Recuperado de http:// www.scielo.org.co/pdf/pys/n45/n45a05.pdf

Arroyo, G. 0. (2013). Soluciones para la baja visión (2a. ed.). Buenos Aires, Argentina: Paratexto. Recuperado de https://www.oftalmologos.org.ar/catalogo/items/ show/4673

Cano, V. (2016). Inclusión a personas con discapacidad visual en los pregrados y licenciaturas en música de Medellín. Recuperado de https://repository.upb.edu.co/ bitstream/handle/20.500.11912/2940/Tesis\%20maestri\%CCC81a\%20en\%20 educacio\%CC\%81n\%20\%20Valen ti\%CC $\% 81$ n\%20Cano\%20Hoyos.pdf?sequence $=1$ 
Cañola, K. (2013). Los docentes deben asumir el reto de la educación inclusiva. Recuperado de http://udep. edu.pe/hoy/2013/los-docentes-deben-asumir-el-reto-de-la-educacion-inclusival

Cast (2008). Universal design for learning guidelines version 1.0. Wakefield, MA.

Delors, J. (1996). Informe a la Unesco de la Comisión Internacional sobre la educación para el siglo xxl: la educación encierra un tesoro. Recuperado de: http://www.unesco. org/education/pdf/DELORS_S.PDF

Fernández, J. M. (2013). Competencias docentes y educación inclusiva. REDIE. Revista Electrónica de Investigación Educativa, 15(2), 82-99.

Galeano, M. E. (2004). Diseño de proyectos de la investigación cualitativa. Medellin, Colombia: Fondo Editorial Eafit.

Gento, P. S., Kvetonová, L. y Rehurek, J. (2011). Tratamiento educativo de la diversidad de tipo visual. Madrid, España: UNED - Universidad Nacional de Educación a Distancia.

Galvis, R. V. (2006). De un perfil docente tradicional a un perfil docente basado en competencias. Acción Pedagógica, 16(1), 48-57.

Gómez, M. R. (2017). Inclusión laboral de docentes con discapacidad visual en el campo educativo. Recuperado de http://repositorio.pedagogica.edu.co/ bitstream/handle/20.500.12209/7812/T021373. pdf? sequence $=1$ ctisAllowed $=y$

Gutiérrez, L. M. (2018). Ficha Informativa de Proyecto. Ministerio de Educación. Recuperado de https://educacion.gob. ec/wp-content/uploads/downloads/2018/04/Proyecto-Modelo-de-Educacion-Inclusiva.pdf

Hernández, M. I. (2015). El Concepto de discapacidad: De la enfermedad al enfoque de derechos. Revista cEs Derecho, 6(2) Julio-diciembre. Recuperado de http://www.scielo. org.co/pdf/cesd/v6n2/v6n2a04.pdf

Jiménez, M. (2017). Las relaciones intersubjetivas como vía de inclusión y construcción del sujeto en la escuela. Nodos y Nudos, 5(43), 77-86. Recuperado de https://doi. org/10.17227/nyn.vol5.num43-6016

Kienyke (2016). Los hermanos invidentes que dan clases en Medellin. Recuperado de https://www.kienyke.com/ historias/los-profesores-invidentes-medellin

Ministerio de Educación (2002). Decreto 1278 de junio 19 de 2002. Recuperado de https://www.mineducacion.gov. co/1621/articles-86102_archivo_pdf.pdf

Ministerio de Educación (2005). Proyecto Educativo Institucional. Recuperado de https://www.mineducacion.gov. co/1621/article-79361.html

Ministerio de Educación (2016a). Resolución N.o 09317 del 06 de mayo de 2016. Recuperado de https://www.mineducacion. gov.co/1759/articles-356906_recurso_1.docx
Ministerio de Educación Nacional (2016b). Banco de la Excelencia. Recuperado de https://bancoexcelencia.mineducacion.gov.co/BancoExcelencia/login.xhtml

Ministerio de Educación (2018). Ley 115 de febrero 8 de 1994. Por la cual se expide la ley general de educación. Recuperado de https://www.mineducacion.gov.co/ 1621/articles-85906_archivo_pdf.pdf

Ministerio de educación, cultura y deporte (2012). Exclusión. Recuperado de: http://www.ite.educacion.es/formacion/ materiales/126/cd/pdf/m3ei.pdf

Martínez, P. C. (2006). El método de estudio de caso. Estrategia metodológica de la investigación científica. Pensamiento \&t Gestión, 20, 165-193. Recuperado de: http:// www.redalyc.org/pdf/646/64 602005.pdf

Moliner, G. O. (2013). Educación inclusiva. Castellón de la Plana. España: Universitat Jaume I. Servei de Comunicació i Publicacions.

Piña de Valderrama, E. (2010). Los significados de la praxis pedagógica en los docentes formadores de formadores. Investigación y Postgrado, mayo-diciembre, 251-272. Recuperado de http://www.redalyc.org/pdf/658/65828402009.pdf

Ramírez, E. (2016). Las prácticas pedagógicas de los maestros y maestras de educación básica primaria que apuntan a la inclusión de estudiantes en situación de discapacidad en el municipio de arboletes. Recuperado de https://repository.upb.edu.co/bitstream/handle/20.500.11912/2865/ TESIS\%20FINAL\%20EULISES\%20RAM\%C3\%8DREZ. pdf?sequence $=1$

RCN (2017). Desempleo de personas con discapacidad visual es siete veces el del pais. Recuperado de https://www.rcnradio. com/economia/desempleo-de-personas-con-discapacidad-visual-es-siete-veces-el-del-pais-segun-estudio

Sandoval, C. A. (1996). Investigación cualitativa. Bogotá, CoIombia: Instituto Colombiano para el Fomento de la Educación Superior, Icfes.

Stenhouse, L. (1991). Investigación y desarrollo del curriculum, Madrid: Ed. Morata.

Sampaio, D. O. A. A., Bortolini, P. R., y Wolf, D. O. F. I. (Eds.). (2014). Prácticas pedagógicas en Educación Especial: hacia una Escuela Inclusiva. Alcalá de Henares, España: Universidad de Alcalá.

Stake, R. (2007). Investigación con estudio de casos. Madrid: Morata.

Taylo, S. J. y Bodgan, R. (1987). Introducción a los métodos cualitativos. Barcelona: Ediciones Paidós.

Unesco (1994). Declaración de Salamanca y marco de acción para las necesidades educativas especiales. Conferencia Mundial Sobre Necesidades Educativas Especiales: acceso y calidad. Salamanca, España, 7-10 de junio. Recuperado de http://www.unesco.org/education/pdf/SALAMA_S.PDF 
Unesco (2006). Orientaciones para la Inclusión: Asegurar el Acceso a la Educación para Todos. Paris.

Vásquez, M. A. (2012). Orientaciones para desarrollar escuelas inclusivas. Recuperado de http://www.seg.guanajuato.
gob.mx/InclusionEducativa/Paginas/materiales/3FUENTES\%20DE\%20CONSULTA/ACADEMICOS/ORIENTACIONES\%2OPARA\%20EL\%20DESARROLLO\%20DE\%20 ESC\%2OINCLUSIVAS.pdf

\section{Anexo A. Matriz metodológica}

\begin{tabular}{|c|c|c|c|c|c|c|}
\hline $\begin{array}{l}\text { Preguntas de } \\
\text { investigación }\end{array}$ & Objetivos & Hipótesis & Metodología & Instrumentos & Variables & Indicadores \\
\hline $\begin{array}{l}\text { ¿Cuáles son las } \\
\text { historias de vida de } \\
\text { los docentes con } \\
\text { discapacidad? }\end{array}$ & $\begin{array}{c}\text { Analizar las } \\
\text { historias de vida de } \\
\text { los docentes con } \\
\text { discapacidad visual. }\end{array}$ & $\begin{array}{l}\text { Al conocer las historias de } \\
\text { cada uno de los docentes que } \\
\text { forman parte del estudio se } \\
\text { logra comprender la realidad } \\
\text { de su experiencia para ser } \\
\text { aplicada en otros docentes de } \\
\text { otras universidades. }\end{array}$ & $\begin{array}{c}\text { Postura } \\
\text { metodológica } \\
\text { de carácter } \\
\text { fenomenológico: } \\
\text { Cualitativa } \\
\text { Estudio de caso. }\end{array}$ & $\begin{array}{l}\text { Entrevista a } \\
\text { profundidad. }\end{array}$ & $\begin{array}{c}\text { Historias } \\
\text { de vida; } \\
\text { Discapacidad; } \\
\text { Significado de } \\
\text { las experiencias. }\end{array}$ & $\begin{array}{c}\text { Cantidad de } \\
\text { aportes. }\end{array}$ \\
\hline $\begin{array}{l}\text { ¿Cómo aporta } \\
\text { la identificación } \\
\text { de los retos y } \\
\text { afrontamientos } \\
\text { personales de } \\
\text { los docentes } \\
\text { desde su rol con } \\
\text { discapacidad } \\
\text { visual? }\end{array}$ & $\begin{array}{l}\text { Identificar los retos } \\
\text { y afrontamientos } \\
\text { personales desde } \\
\text { el rol docente con } \\
\text { discapacidad visual. }\end{array}$ & $\begin{array}{l}\text { Conociendo los retos que } \\
\text { afrontan los docentes en } \\
\text { situación de discapacidad a } \\
\text { nivel personal para brindar } \\
\text { su servicio contribuye a } \\
\text { la generación de cambio } \\
\text { e inserción de nuevas } \\
\text { politicas de inclusión en las } \\
\text { instituciones educativas. }\end{array}$ & $\begin{array}{l}\text { Cualitativa; } \\
\text { estudio de caso. }\end{array}$ & $\begin{array}{l}\text { Entrevista a } \\
\text { profundidad. }\end{array}$ & $\begin{array}{l}\text { Retos } \\
\text { personales; } \\
\text { Afrontamientos } \\
\text { personales; Rol } \\
\text { del docente }\end{array}$ & $\begin{array}{c}\text { Cantidad de } \\
\text { aportes. }\end{array}$ \\
\hline $\begin{array}{l}\text { ¿Cuáles son } \\
\text { las actitudes y } \\
\text { aptitudes de los } \\
\text { docentes con } \\
\text { discapacidad } \\
\text { visual que aportan } \\
\text { positivamente en la } \\
\text { educación inclusiva } \\
\text { en Santander? }\end{array}$ & $\begin{array}{c}\text { Reconocer } \\
\text { actitudes y } \\
\text { aptitudes de los } \\
\text { docentes con } \\
\text { discapacidad } \\
\text { visual que aportan } \\
\text { positivamente en la } \\
\text { educación inclusiva } \\
\text { en Santander. }\end{array}$ & $\begin{array}{c}\text { Cuando los docentes con } \\
\text { discapacidad visual poseen } \\
\text { actitudes yaptitudes } \\
\text { positivas se preparan para } \\
\text { asumir todo lo que conlleva } \\
\text { la educación inclusiva, } \\
\text { adaptaciones, currículo, } \\
\text { material, flexibilidad, } \\
\text { permanecía, actualización } \\
\text { profesional, entre otras. }\end{array}$ & $\begin{array}{c}\text { Cualitativa; } \\
\text { estudio de caso. }\end{array}$ & $\begin{array}{l}\text { Entrevista a } \\
\text { profundidad }\end{array}$ & $\begin{array}{l}\text { Aptitudes y } \\
\text { actitudes; } \\
\text { educación } \\
\text { inclusiva. }\end{array}$ & $\begin{array}{c}\text { Cantidad de } \\
\text { aportes. }\end{array}$ \\
\hline
\end{tabular}




\section{Diálogo del conocimiento}

Al abordar la comprensión sobre las condiciones y características del ejercicio docente de maestras y maestros con discapacidad, en este caso con discapacidad visual, el artículo presenta un desarrollo investigativo original en la reflexión y en la investigación sobre la educación inclusiva en Colombia. Existen diferentes trabajos sobre las implicaciones de la discapacidad en el aprendizaje y, en especial, sobre diferentes maneras de superar las barreras que se presentan para la garantía del derecho a la educación de estudiantes con discapacidad, en especial para su acceso y permanencia en el sistema educativo, así como para sus procesos de aprendizaje y para sus formas de participación de la vida escolar. Sin embargo, son prácticamente inexistentes miradas investigativas sobre el desempeño y sobre las formas de ser maestro de profesoras y profesores con discapacidad.

Es también destacable que la investigación que fundamenta el artículo se desarrolla con cinco maestros y maestras con discapacidad visual que trabajan en sectores rurales del departamento de Santander, a partir de la construcción o el desarrollo de sus historias de vida. Este contexto de ruralidad también constituye un aporte innovador del artículo, pues no son muy comunes las exploraciones e indagaciones sobre educación inclusiva en sectores rurales del país. La mayoría de las aproximaciones y de los estudios sobre prácticas y procesos de educación inclusiva con estudiantes con discapacidad centran sus miradas en colegios y en experiencias del sector urbano.

Rafael Pabón García Consultor independiente en educación rafael.pabon.g@gmail.com 